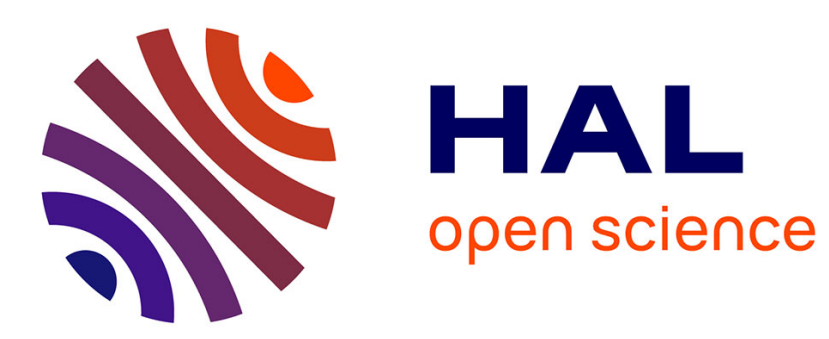

\title{
An approximation method for exact controls of vibrating systems
}

Nicolae Cindea, Sorin Micu, Marius Tucsnak

\section{To cite this version:}

Nicolae Cindea, Sorin Micu, Marius Tucsnak. An approximation method for exact controls of vibrating systems. SIAM Journal on Control and Optimization, 2011, 49 (3), pp.1283-1305. 10.1137/09077641X . hal-00430266

\section{HAL Id: hal-00430266 https://hal.science/hal-00430266}

Submitted on 6 Nov 2009

HAL is a multi-disciplinary open access archive for the deposit and dissemination of scientific research documents, whether they are published or not. The documents may come from teaching and research institutions in France or abroad, or from public or private research centers.
L'archive ouverte pluridisciplinaire HAL, est destinée au dépôt et à la diffusion de documents scientifiques de niveau recherche, publiés ou non, émanant des établissements d'enseignement et de recherche français ou étrangers, des laboratoires publics ou privés. 


\title{
An approximation method for exact controls of vibrating systems
}

\author{
Nicolae Cîndea \\ Institut Elie Cartan, Nancy Université/CNRS/INRIA \\ BP 70239, 54506 Vandoeuvre-lès-Nancy, France \\ cindea@iecn.u-nancy.fr \\ Sorin Micu \\ Department of Mathematics, University of Craiova \\ Craiova, 200585, Romania \\ sd_micu@yahoo.com \\ Marius Tucsnak \\ Institut Elie Cartan, Nancy Université/CNRS/INRIA \\ BP 70239, 54506 Vandoeuvre-lès-Nancy, France \\ Marius. Tucsnak@iecn.u-nancy.fr
}

\begin{abstract}
We propose a new method for the approximation of exact controls of a second order infinite dimensional system with bounded input operator. The algorithm combines Russell's "stabilizability implies controllability" principle with the Galerkin's method. The main new feature brought in by this work consists in giving precise error estimates. In order to test the efficiency of the method, we consider two illustrative examples (with the finite element approximations of the wave and the beam equations) and we describe the corresponding simulations.
\end{abstract}

Mathematics Subject Classification (2000): 35L10, 65M60, 93B05, 93B40, 93D15.

Key words: infinite dimensional systems, exact control, approximation, error estimate.

\section{Introduction}

The numerical study of the exact controls of infinite dimensional systems started in the 90's with a series of papers of Glowinski and Lions (see [7, 8]) where algorithms to determine the minimal $L^{2}$-norm exact controls (sometimes called HUM controls) are provided. Several abnormalities presented in these works stand at the origin of a large number of articles in which a great variety of numerical methods are presented and analyzed (see, for instance, [21], [6] and the references therein). However, except the recent work [5], where the approximation of the HUM controls for the one dimensional wave equation is considered, to our knowledge, there are no results on the rate of convergence of the approximative controls. 
The aim of this work is to provide an efficient numerical method for computing exact controls for a class of infinite dimensional systems modeling elastic vibrations. Our main theoretical result gives the rate of convergence of our approximations to an exact control. Moreover, to illustrate the efficiency of this approach, we apply it to several systems governed by PDE's and we describe the associated numerical simulations. Our methodology combines Russell's "stabilizability implies controllability" principle with error estimates for finite element type approximations of the considered infinite dimensional systems. We focus on the case of bounded input operators which excludes boundary control for systems governed by partial differential equations. However, the method can be partially extended to the unbounded input operator case, see Remark 2.7 below.

In order to give the precise statement of our results we need some notation. Let $H$ be a Hilbert space and assume that $A_{0}: \mathcal{D}\left(A_{0}\right) \rightarrow H$ is a self-adjoint, strictly positive operator with compact resolvents. Then, according to classical results, the operator $A_{0}$ is diagonalizable with an orthonormal basis $\left(\varphi_{k}\right)_{k} \geqslant 1$ of eigenvectors and the corresponding family of positive eigenvalues $\left(\lambda_{k}\right)_{k \geqslant 1}$ satisfies $\lim _{k \rightarrow \infty} \lambda_{k}=\infty$. Moreover, we have

$$
\mathcal{D}\left(A_{0}\right)=\left\{\left.z \in H\left|\sum_{k \geqslant 1} \lambda_{k}^{2}\right|\left\langle z, \varphi_{k}\right\rangle\right|^{2}<\infty\right\},
$$

and

$$
A_{0} z=\sum_{k \geqslant 1} \lambda_{k}\left\langle z, \varphi_{k}\right\rangle \varphi_{k} \quad\left(z \in \mathcal{D}\left(A_{0}\right)\right) .
$$

For $\alpha \geqslant 0$ the operator $A_{0}^{\alpha}$ is defined by

$$
\mathcal{D}\left(A_{0}^{\alpha}\right)=\left\{\begin{array}{l|l}
z \in H & \sum_{k \geqslant 1} \lambda_{k}^{2 \alpha}\left|\left\langle z, \varphi_{k}\right\rangle\right|^{2}<\infty
\end{array}\right\},
$$

and

$$
A_{0}^{\alpha} z=\sum_{k \geqslant 1} \lambda_{k}^{\alpha}\left\langle z, \varphi_{k}\right\rangle \varphi_{k} \quad\left(z \in \mathcal{D}\left(A_{0}^{\alpha}\right)\right) .
$$

For every $\alpha \geqslant 0$ we denote by $H_{\alpha}$ the space $\mathcal{D}\left(A_{0}^{\alpha}\right)$ endowed with the inner product

$$
\langle\varphi, \psi\rangle_{\alpha}=\left\langle A_{0}^{\alpha} \varphi, A_{0}^{\alpha} \psi\right\rangle \quad\left(\varphi, \psi \in H_{\alpha}\right) .
$$

The induced norm is denoted by $\|\cdot\|_{\alpha}$. From the above facts it follows that for every $\alpha \geqslant 0$ the operator $A_{0}$ is a unitary operator from $H_{\alpha+1}$ onto $H_{\alpha}$ and $A_{0}$ is strictly positive on $H_{\alpha}$.

Let $U$ be another Hilbert space and let $B_{0} \in \mathcal{L}(U, H)$ be an input operator. Consider the system

$$
\begin{gathered}
\ddot{q}(t)+A_{0} q(t)+B_{0} u(t)=0 \quad(t \geqslant 0), \\
q(0)=q_{0}, \dot{q}(0)=q_{1} .
\end{gathered}
$$

The above system is said exactly controllable in time $\tau>0$ if for every $q_{0} \in H_{\frac{1}{2}}, q_{1} \in H$ there exists a control $u \in L^{2}([0, \tau], U)$ such that $q(\tau)=\dot{q}(\tau)=0$. In order to provide a numerical method to approximate such a control $u$, we need more assumptions and notation. 
Assume that there exists family $\left(V_{h}\right)_{h>0}$ of finite dimensional subspaces of $H_{\frac{1}{2}}$ and that there exist $\theta>0, h^{*}>0, C_{0}>0$ such that, for every $h \in\left(0, h^{*}\right)$,

$$
\begin{aligned}
& \left\|\pi_{h} \varphi-\varphi\right\|_{\frac{1}{2}} \leq C_{0} h^{\theta}\|\varphi\|_{1} \quad\left(\varphi \in H_{1}\right), \\
& \left\|\pi_{h} \varphi-\varphi\right\| \leq C_{0} h^{\theta}\|\varphi\|_{\frac{1}{2}} \quad\left(\varphi \in H_{\frac{1}{2}}\right),
\end{aligned}
$$

where $\pi_{h}$ is the orthogonal projector from $H_{\frac{1}{2}}$ onto $V_{h}$. Assumptions (1.4)-(1.5) are, in particular, satisfied when finite elements are used for the approximation of Sobolev spaces. The inner product in $V_{h}$ is the restriction of the inner product on $H$ and it is still denoted by $\langle\cdot, \cdot\rangle$. We define the linear operator $A_{0 h} \in \mathcal{L}\left(V_{h}\right)$ by

$$
\left\langle A_{0 h} \varphi_{h}, \psi_{h}\right\rangle=\left\langle A_{0}^{\frac{1}{2}} \varphi_{h}, A_{0}^{\frac{1}{2}} \psi_{h}\right\rangle \quad\left(\varphi_{h}, \psi_{h} \in V_{h}\right) .
$$

The operator $A_{0 h}$ is clearly symmetric and strictly positive.

Denote $U_{h}=B_{0}^{*} V_{h} \subset U$ and define the operators $B_{0 h} \in \mathcal{L}(U, H)$ by

$$
B_{0 h} u=\tilde{\pi}_{h} B_{0} u \quad(u \in U),
$$

where $\tilde{\pi}_{h}$ is the orthogonal projection of $H$ onto $V_{h}$. Note that $\operatorname{Ran} B_{0 h} \subset V_{h}$. As wellknown, since it is a projector, the operator $\widetilde{\pi}_{h} \in \mathcal{L}(H)$ is self-adjoint. Moreover, from (1.5) we deduce that

$$
\left\|\varphi-\widetilde{\pi}_{h} \varphi\right\| \leqslant\left\|\varphi-\pi_{h} \varphi\right\| \leqslant C_{0} h^{\theta}\|\varphi\|_{\frac{1}{2}} \quad\left(\varphi \in H_{\frac{1}{2}}\right) .
$$

The adjoint $B_{0 h}^{*} \in \mathcal{L}(H, U)$ of $B_{0 h}$ is

$$
B_{0 h}^{*} \varphi=B_{0}^{*} \tilde{\pi}_{h} \varphi \quad(\varphi \in H) .
$$

Since $U_{h}=B_{0}^{*} V_{h}$, from (1.9) it follows that $\operatorname{Ran} B_{0 h}^{*}=U_{h}$ and that

$$
\left\langle B_{0 h}^{*} \varphi_{h}, B_{0 h}^{*} \psi_{h}\right\rangle_{U}=\left\langle B_{0}^{*} \varphi_{h}, B_{0}^{*} \psi_{h}\right\rangle_{U} \quad\left(\varphi_{h}, \psi_{h} \in V_{h}\right) .
$$

The above assumptions imply that, for every $h^{*}>0$, the family $\left(\left\|B_{0 h}\right\|_{\mathcal{L}(U, H)}\right)_{h \in\left(0, h^{*}\right)}$ is bounded.

In what follows, we give an algorithm to compute an approximation $u_{h} \in C\left([0, \tau] ; U_{h}\right)$ of an exact control $u \in C([0, \tau] ; U)$, which drives the solution of (1.2)-(1.3) from the initial state $\left[\begin{array}{c}q_{0} \\ q_{1}\end{array}\right] \in H_{1} \times H_{\frac{1}{2}}$ to rest in time $\tau$. For every $h>0$ and $n \geqslant 1$ we consider the following second order ODE's :

$$
\begin{gathered}
\ddot{w}_{h}^{n}(t)+A_{0 h} w_{h}^{n}(t)+B_{0 h} B_{0 h}^{*} \dot{w}_{h}^{n}(t)=0 \quad(t \geqslant 0) \\
w_{h}^{n}(0)=\left\{\begin{array}{ll}
\pi_{h} q_{0}, & \text { if } n=1 \\
w_{b, h}^{n-1}(0), & \text { if } n>1
\end{array} \quad \dot{w}_{h}^{n}(0)= \begin{cases}\pi_{h} q_{1}, & \text { if } n=1 \\
\dot{w}_{b, h}^{n-1}(0), & \text { if } n>1,\end{cases} \right.
\end{gathered}
$$

and

$$
\begin{gathered}
\ddot{w}_{b, h}^{n}(t)+A_{0 h} w_{b, h}^{n}(t)-B_{0 h} B_{0 h}^{*} \dot{w}_{b, h}^{n}(t)=0 \quad(t \leqslant \tau) \\
w_{b, h}^{n}(\tau)=w_{h}^{n}(\tau), \quad \dot{w}_{b, h}^{n}(\tau)=\dot{w}_{h}^{n}(\tau) .
\end{gathered}
$$


For every $h>0$ we appropriately choose $N(h) \in \mathbb{N}$ (see Theorem 1.1 below) and we define

$$
\left[\begin{array}{c}
w_{0 h} \\
w_{1 h}
\end{array}\right]=\left[\begin{array}{c}
\pi_{h} q_{0} \\
\pi_{h} q_{1}
\end{array}\right]+\sum_{n=1}^{N(h)}\left[\begin{array}{c}
w_{b, h}^{n}(0) \\
\dot{w}_{b, h}^{n}(0)
\end{array}\right]
$$

With this notation, $u_{h}$ is defined by

$$
u_{h}=B_{0 h}^{*} \dot{w}_{h}+B_{0 h}^{*} \dot{w}_{b, h},
$$

where $w_{h}$ and $w_{b, h}$ are the solution of

$$
\begin{gathered}
\ddot{w}_{h}(t)+A_{0 h} w_{h}(t)+B_{0 h} B_{0 h}^{*} \dot{w}_{h}(t)=0 \quad(t \geqslant 0) \\
w_{h}(0)=w_{0 h}, \quad \dot{w}_{h}(0)=w_{1 h}, \\
\ddot{w}_{b, h}(t)+A_{0 h} w_{b, h}(t)-B_{0 h} B_{0 h}^{*} \dot{w}_{b, h}(t)=0 \quad(t \leqslant \tau) \\
w_{b, h}(\tau)=w_{h}(\tau), \quad \dot{w}_{b, h}(\tau)=\dot{w}_{h}(\tau) .
\end{gathered}
$$

We can now formulate the main result of this paper.

Theorem 1.1. With the above notation and assumptions, assume furthermore that the system (1.2), (1.3) is exactly controllable in some time $\tau>0$ and that $B_{0} B_{0}^{*} \in \mathcal{L}\left(H_{1}, H_{\frac{1}{2}}\right)$. Then there exists a constant $m_{\tau}>0$ such that the sequence $\left(u_{h}\right)_{h \geqslant 0}$ of $C\left([0, \tau] ; U_{h}\right)$ defined in (1.16) with $N(h)=\left[\theta m_{\tau} \ln \left(h^{-1}\right)\right]$, converges when $h \rightarrow 0$ to an exact control in time $\tau$ of (1.2), (1.3), denoted by $u$, for every $Q_{0}=\left[\begin{array}{l}q_{0} \\ q_{1}\end{array}\right] \in H_{\frac{3}{2}} \times H_{1}$. Moreover, there exist constants $h^{*}>0$ and $C:=C_{\tau}$ such that we have

$$
\left\|u-u_{h}\right\|_{C([0, \tau] ; U)} \leqslant C h^{\theta} \ln ^{2}\left(h^{-1}\right)\left\|Q_{0}\right\|_{H_{\frac{3}{2}} \times H_{1}} \quad\left(0<h<h^{*}\right) .
$$

We prove this theorem in Section 4. In the second section we recall some background on exact controllability and stabilizability. Section 3 provides some error estimates. In Section 5 we apply our results to the wave equation in two space dimensions and to the Euler-Bernoulli beam equation, providing numerical simulations.

\section{Some background on exact controllability and uniform stabilization}

In this section we recall, with no claim of originality, some background concerning the exact controllability and uniform stabilizability of the system (1.2), (1.3). We give, in particular, a short proof, adapted to our case, of Russell's "stabilizability implies controllability" principle. This principle has been originally stated in Russell [15, 16] (see also Chen [4]).

Consider the second order differential equation

$$
\begin{gathered}
\ddot{w}(t)+A_{0} w(t)+B_{0} B_{0}^{*} \dot{w}(t)=0 \quad(t \geqslant 0), \\
w(0)=w_{0}, \dot{w}(0)=w_{1} .
\end{gathered}
$$

It is well known that the above equation defines a well posed dynamical system in the state space $X=H_{\frac{1}{2}} \times H$. More precisely, the solution $\left[\begin{array}{c}w \\ w\end{array}\right]$ of $(2.1),(2.2)$ is given by

$$
\left[\begin{array}{l}
w(t) \\
\dot{w}(t)
\end{array}\right]=\mathbb{T}_{t}\left[\begin{array}{l}
w_{0} \\
w_{1}
\end{array}\right] \quad\left(\left[\begin{array}{l}
w_{0} \\
w_{1}
\end{array}\right] \in X, \quad t \geqslant 0\right)
$$


where $\mathbb{T}$ is the contraction semigroup on $X$ generated by $\mathcal{A}-\mathcal{B B}^{*}$ and $\mathcal{A}: \mathcal{D}(\mathcal{A}) \rightarrow X$, $\mathcal{B} \in \mathcal{L}(U, X)$ are defined by

$$
\mathcal{D}(\mathcal{A})=H_{1} \times H_{\frac{1}{2}}, \quad \mathcal{A}=\left[\begin{array}{cc}
0 & I \\
-A_{0} & 0
\end{array}\right], \quad \mathcal{B}=\left[\begin{array}{c}
0 \\
B_{0}
\end{array}\right] .
$$

We also consider the backwards system

$$
\begin{gathered}
\ddot{w}_{b}(t)+A_{0} w_{b}(t)-B_{0} B_{0}^{*} \dot{w}_{b}(t)=0 \quad(t \leqslant \tau), \\
w_{b}(\tau)=w(\tau), \dot{w}_{b}(\tau)=\dot{w}(\tau) .
\end{gathered}
$$

It is not difficult to check that the solution $\left[\begin{array}{l}w_{b} \\ w_{b}\end{array}\right]$ of $(2.4),(2.5)$ is given by

$$
\left[\begin{array}{c}
w_{b}(t) \\
\dot{w}_{b}(t)
\end{array}\right]=\mathbb{S}_{\tau-t}\left[\begin{array}{c}
w(\tau) \\
\dot{w}(\tau)
\end{array}\right] \quad(t \in[0, \tau]),
$$

where $\mathbb{S}$ is the contraction semigroup in $X$ generated by $-\mathcal{A}-\mathcal{B B}^{*}$.

We define $L_{\tau} \in \mathcal{L}(X)$ by

$$
L_{\tau}\left[\begin{array}{l}
w_{0} \\
w_{1}
\end{array}\right]=\left[\begin{array}{l}
w_{b}(0) \\
\dot{w}_{b}(0)
\end{array}\right] \quad\left(\left[\begin{array}{l}
w_{0} \\
w_{1}
\end{array}\right] \in X\right) .
$$

With the above notation, the operator $L_{\tau}$ clearly satisfies $L_{\tau}=\mathbb{S}_{\tau} \mathbb{T}_{\tau}$.

Proposition 2.1. With the above notation, assume that the system (1.2), (1.3) is exactly controllable in some time $\tau>0$. Then the semigroups $\mathbb{T}$ and $\mathbb{S}$ are exponentially stable and we have $\left\|\mathbb{T}_{\tau}\right\|_{\mathcal{L}(X)}<1$ and $\left\|\mathbb{S}_{\tau}\right\|_{\mathcal{L}(X)}<1$. Moreover, the operator $I-L_{\tau}$ is invertible and we have

$$
\left(I-L_{\tau}\right)^{-1}=\sum_{n \geqslant 0} L_{\tau}^{n}
$$

Proof. The fact that $\mathbb{T}$ and $\mathbb{S}$ are exponentially stable is well-known (see, for instance, Haraux [9] and Liu [12]). The more precise facts that $\left\|\mathbb{T}_{\tau}\right\|_{\mathcal{L}(X)}<1$ and $\left\|\mathbb{S}_{\tau}\right\|_{\mathcal{L}(X)}<1$ are easy to establish (see, for instance, Lemma 2.2 in Ito, Ramdani and Tucsnak [10]). Finally, (2.8) follows from $\left\|L_{\tau}\right\|_{\mathcal{L}(X)}<1$.

The particular case of Russell's principle [16], which we need in this work, is given by the following result:

Proposition 2.2. Assume that (1.2), (1.3) is exactly controllable in time $\tau>0$. Then a control $u \in C([0, \tau] ; U)$ for $(1.2),(1.3)$ steering the initial state $\left[\begin{array}{c}q_{0} \\ q_{1}\end{array}\right] \in X$ to rest in time $\tau$ is given by

$$
u=B_{0}^{*} \dot{w}+B_{0}^{*} \dot{w}_{b}
$$

where $w$ and $w_{b}$ are the solutions of (2.1)-(2.2) and (2.4)-(2.5) respectively, with

$$
\left[\begin{array}{l}
w_{0} \\
w_{1}
\end{array}\right]=\left(I-L_{\tau}\right)^{-1}\left[\begin{array}{l}
q_{0} \\
q_{1}
\end{array}\right] .
$$


Remark 2.3. The original assumption of Russell's principle was essentially the exponential stability of the semigroups $\mathbb{T}$ and $\mathbb{S}$, whence the name "stabilizability implies controllability". Since, according to Proposition 2.1, these stability properties are equivalent to the exact controllability of (1.2), (1.3), we made this assumption explicitly in Proposition 2.2. The essential thing retained from the original Russell's principle is the specific form (2.9) of u, obtained using the "closed loop semigroups" $\mathbb{T}$ and $\mathbb{S}$.

Proof of Proposition 2.2. Denote

$$
q(t)=w(t)-w_{b}(t) \quad(t \in[0, \tau]) .
$$

Then $q$ clearly satisfies (1.2) with $u$ given by (2.9). Moreover, from (2.10) it follows that $q$ satisfies the initial conditions (1.3). Finally, from (2.5) it follows that

$$
q(\tau)=\dot{q}(\tau)=0
$$

Remark 2.4. Using the semigroup notation, an alternative way of writing (2.9) is

$$
u(t)=\mathcal{B}^{*} \mathbb{T}_{t}\left[\begin{array}{l}
w_{0} \\
w_{1}
\end{array}\right]+\mathcal{B}^{*} \mathbb{S}_{\tau-t} \mathbb{T}_{\tau}\left[\begin{array}{l}
w_{0} \\
w_{1}
\end{array}\right] \quad(t \in[0, \tau]),
$$

where $w_{0}, w_{1}$ satisfy $(2.10)$.

We need below the fact that the restrictions of $\mathbb{T}$ and $\mathbb{S}$ to $H_{1} \times H_{\frac{1}{2}}$ and $H_{\frac{3}{2}} \times H_{1}$ are exponentially stable semigroups. Sufficient conditions for this are given in the result below.

Proposition 2.5. Assume that $B_{0} B_{0}^{*} \in \mathcal{L}\left(H_{1}, H_{\frac{1}{2}}\right)$. Then the restrictions of $\mathbb{T}$ and $\mathbb{S}$ to $H_{1} \times H_{\frac{1}{2}}$ and $H_{\frac{3}{2}} \times H_{1}$ are contraction semigroups on these spaces with generators the restrictions of $\mathcal{A}-\mathcal{B B}^{*}$ and $-\mathcal{A}-\mathcal{B B}^{*}$ to $H_{\frac{3}{2}} \times H_{1}$ and $H_{2} \times H_{\frac{3}{2}}$ respectively. Moreover, if (1.2), (1.3) is exactly controllable in time $\tau>0$ then

$$
\left\|\mathbb{T}_{\tau}\right\|_{\mathcal{L}\left(H_{\frac{3}{2}} \times H_{1}\right)}<1, \quad\left\|\mathbb{S}_{\tau}\right\|_{\mathcal{L}\left(H_{\frac{3}{2}} \times H_{1}\right)}<1 .
$$

Proof. From a well-known result (see, for instance, [19, Proposition 2.10.4]) it follows that the restriction of $\mathbb{T}$ to $\mathcal{D}\left(\mathcal{A}-\mathcal{B B}^{*}\right)=H_{1} \times H_{\frac{1}{2}}$ is a contraction semigroup on $\mathcal{D}\left(\mathcal{A}-\mathcal{B B}^{*}\right)$ (endowed with the graph norm) whose generator is the restriction of $\mathcal{A}-\mathcal{B B}^{*}$ to $\mathcal{D}((\mathcal{A}-$ $\left.\left.\mathcal{B B}^{*}\right)^{2}\right)$. Since $B_{0} B_{0}^{*} \in \mathcal{L}\left(H_{1}, H_{\frac{1}{2}}\right)$, it is easy to check that $\mathcal{D}\left(\left(\mathcal{A}-\mathcal{B B}^{*}\right)^{2}\right)=H_{\frac{3}{2}} \times H_{1}$. It follows that indeed the restriction of $\mathbb{T}$ to $H_{1} \times H_{\frac{1}{2}}$ is a contraction semigroup on this space with generator the restriction of $\mathcal{A}-\mathcal{B B}^{*}$ to $H_{\frac{3}{2}} \times H_{1}$. The second assertion on $\mathbb{T}$ can be easily obtained by iterating this argument. The corresponding assertions for $\mathbb{S}$ can be proved in a completely similar manner. Finally, the estimates (2.12) follow from the corresponding estimates for the norms in $\mathcal{L}\left(H_{1} \times H_{\frac{1}{2}}\right)$ (see Proposition 2.1) by applying again Proposition 2.10.4 in [19].

Remark 2.6. An important property of the control u constructed in (1.16) is that, under appropriate assumptions on $B_{0}$, its regularity increases when the initial data are more regular. For instance, if $B_{0} B_{0}^{*} \in \mathcal{L}\left(H_{1}, H_{\frac{1}{2}}\right)$ and $\left[\begin{array}{c}q_{0} \\ q_{1}\end{array}\right] \in H_{\frac{3}{2}} \times H_{1}$ then, by Proposition 2.5, $\left[\begin{array}{c}w_{0} \\ w_{1}\end{array}\right]=\left(I-L_{\tau}\right)^{-1}\left[\begin{array}{c}q_{0} \\ q_{1}\end{array}\right] \in H_{\frac{3}{2}} \times H_{1}$ so that $u \in C^{1}([0, \tau] ; U)$ and $B_{0} u \in C\left([0, \tau] ; H_{\frac{1}{2}}\right)$. 
Remark 2.7. Russell's principle can be extended to the the case of unbounded input operators $B_{0} \in \mathcal{L}\left(U, H_{-\frac{1}{2}}\right)$, where $H_{-\frac{1}{2}}$ is the dual of $H_{\frac{1}{2}}$ with respect to the pivot space $H$. In this case the system (2.1)-(2.2) is still well-posed and it keeps most of the properties holding for bounded $B_{0}$ (see, for instance, [20], [18] and references therein). For a quite general form of Russell's principle for unbounded input operators we refer to [14].

\section{An approximation result}

The aim of this section is to provide error estimates for the approximations of (2.1) by finite-dimensional systems. Using the notation in Section 1 for the families of spaces $\left(V_{h}\right)_{h>0},\left(U_{h}\right)_{h>0}$ and the families of operators $\left(\pi_{h}\right)_{h>0},\left(A_{0 h}\right)_{h>0},\left(B_{0 h}\right)_{h>0}$, we consider the family of finite dimensional systems

$$
\begin{gathered}
\ddot{w}_{h}(t)+A_{0 h} w_{h}(t)+B_{0 h} B_{0 h}^{*} \dot{w}_{h}(t)=0, \\
w_{h}(0)=\pi_{h} w_{0}, \quad \dot{w}_{h}(0)=\pi_{h} w_{1} .
\end{gathered}
$$

In the case in which $B_{0}=0$ and $A_{0}$ is the Dirichlet Laplacian, it has been shown in Baker [1] that, given $w_{0} \in H_{\frac{3}{2}}, w_{1} \in H_{1}$, the solutions of (3.1) converge when $h \rightarrow 0$ to the solution of (2.1). Moreover, [1] contains precise estimates of the convergence rate. The result below shows that the same error estimates hold when $A_{0}$ is an arbitrary positive operator and $B_{0} \neq 0$. Throughout this section we assume that $B_{0} B_{0}^{*} \in \mathcal{L}\left(H_{1}, H_{\frac{1}{2}}\right)$.

Proposition 3.1. Let $w_{0} \in H_{\frac{3}{2}}, w_{1} \in H_{1}$ and let $w, w_{h}$ be the corresponding solutions of (2.1), (2.2) and (3.1), (3.2). Moreover, assume that $B_{0} B_{0}^{*} \in \mathcal{L}\left(H_{1}, H_{\frac{1}{2}}\right)$. Then there exist three constants $K_{0}, K_{1}, h^{*}>0$ such that, for every $h \in\left(0, h^{*}\right)$, we have

$$
\left\|\dot{w}(t)-\dot{w}_{h}(t)\right\|+\left\|w(t)-w_{h}(t)\right\|_{\frac{1}{2}} \leqslant\left(K_{0}+K_{1} t\right) h^{\theta}\left(\left\|w_{0}\right\|_{\frac{3}{2}}+\left\|w_{1}\right\|_{1}\right) \quad(t \geqslant 0) .
$$

Proof. We first note that, according to Proposition 2.5, we have

$$
\begin{gathered}
w \in C\left([0, \infty) ; H_{\frac{3}{2}}\right) \cap C^{1}\left([0, \infty) ; H_{1}\right) \cap C^{2}\left([0, \infty) ; H_{\frac{1}{2}}\right), \\
\|\ddot{w}(t)\|_{\frac{1}{2}}+\|\dot{w}(t)\|_{1}+\|w(t)\|_{\frac{3}{2}} \leqslant\left\|w_{1}\right\|_{1}+\left\|w_{0}\right\|_{\frac{3}{2}} \quad(t \geqslant 0) .
\end{gathered}
$$

Equation (2.1) can be written

$$
\langle\ddot{w}, v\rangle+\left\langle A_{0}^{\frac{1}{2}} w, A_{0}^{\frac{1}{2}} v\right\rangle+\left\langle B_{0}^{*} \dot{w}, B_{0}^{*} v\right\rangle_{U}=0 \quad\left(v \in H_{\frac{1}{2}}\right),
$$

whereas, using (1.6) and (1.10), we see that (3.1) is equivalent to

$$
\left\langle\ddot{w}_{h}, v_{h}\right\rangle+\left\langle A_{0}^{\frac{1}{2}} w_{h}, A_{0}^{\frac{1}{2}} v_{h}\right\rangle+\left\langle B_{0}^{*} \dot{w}_{h}, B_{0}^{*} v_{h}\right\rangle_{U}=0 \quad\left(v_{h} \in V_{h}\right) .
$$

Taking $v=v_{h}$ in the first one of the above relations and subtracting side by side it follows that

$$
\left\langle\ddot{w}-\ddot{w}_{h}, v_{h}\right\rangle+\left\langle A_{0}^{\frac{1}{2}}\left(w-w_{h}\right), A_{0}^{\frac{1}{2}} v_{h}\right\rangle+\left\langle B_{0}^{*} \dot{w}-B_{0}^{*} \dot{w}_{h}, B_{0}^{*} v_{h}\right\rangle_{U}=0 \quad\left(v_{h} \in V_{h}\right)
$$


which yields (recall that $\pi_{h}$ is the orthogonal projector from $H_{\frac{1}{2}}$ onto $V_{h}$ ) that

$$
\begin{aligned}
\left\langle\pi_{h} \ddot{w}-\ddot{w}_{h}, v_{h}\right\rangle+\left\langle A_{0}^{\frac{1}{2}}\left(\pi_{h} w-w_{h}\right), A_{0}^{\frac{1}{2}} v_{h}\right\rangle & \\
& =\left\langle\pi_{h} \ddot{w}-\ddot{w}, v_{h}\right\rangle-\left\langle B_{0}^{*} \dot{w}-B_{0}^{*} \dot{w}_{h}, B_{0}^{*} v_{h}\right\rangle_{U} \quad\left(v_{h} \in V_{h}\right) .
\end{aligned}
$$

We set

$$
\mathcal{E}_{h}(t)=\frac{1}{2}\left\|\pi_{h} \dot{w}-\dot{w}_{h}\right\|^{2}+\frac{1}{2}\left\|A_{0}^{\frac{1}{2}}\left(\pi_{h} w-w_{h}\right)\right\|^{2} .
$$

Using (3.5) it follows that

$$
\begin{aligned}
\dot{\mathcal{E}}_{h}(t) & =\left\langle\pi_{h} \ddot{w}-\ddot{w}, \pi_{h} \dot{w}-\dot{w}_{h}\right\rangle-\left\langle B_{0}^{*}\left(\dot{w}-\dot{w}_{h}\right), B_{0}^{*}\left(\pi_{h} \dot{w}-\dot{w}_{h}\right)\right\rangle_{U} \\
& =\left\langle\pi_{h} \ddot{w}-\ddot{w}, \pi_{h} \dot{w}-\dot{w}_{h}\right\rangle-\left\|B_{0}^{*}\left(\pi_{h} \dot{w}-\dot{w}_{h}\right)\right\|_{U}^{2}+\left\langle B_{0} B_{0}^{*}\left(\pi_{h} \dot{w}-\dot{w}\right),\left(\pi_{h} \dot{w}-\dot{w}_{h}\right)\right\rangle .
\end{aligned}
$$

We have thus shown that

$$
\dot{\mathcal{E}}_{h}(t) \leqslant M\left(\left\|\pi_{h} \ddot{w}-\ddot{w}\right\|+\left\|\pi_{h} \dot{w}-\dot{w}\right\|\right)\left\|\pi_{h} \dot{w}-\dot{w}_{h}\right\|,
$$

where $M=1+\left\|B_{0} B_{0}^{*}\right\|$. It follows that

$$
2 \mathcal{E}_{h}^{\frac{1}{2}}(t) \frac{\mathrm{d}}{\mathrm{d} t} \mathcal{E}_{h}^{\frac{1}{2}}(t) \leqslant M \sqrt{2}\left(\left\|\pi_{h} \ddot{w}-\ddot{w}\right\|+\left\|\pi_{h} \dot{w}-\dot{w}\right\|\right) \mathcal{E}_{h}^{\frac{1}{2}}(t),
$$

which yields

$$
\mathcal{E}_{h}^{\frac{1}{2}}(t) \leqslant \mathcal{E}_{h}^{\frac{1}{2}}(0)+M \sqrt{2} \int_{0}^{t}\left(\left\|\pi_{h} \ddot{w}-\ddot{w}\right\|+\left\|\pi_{h} \dot{w}-\dot{w}\right\|\right) \mathrm{d} t \quad(t \geqslant 0) .
$$

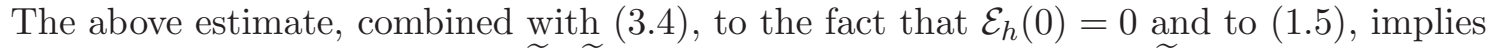
that there exist two constants $\widetilde{K}, \widetilde{h}^{*}>0$ such that, for every $h \in\left(0, \widetilde{h}^{*}\right)$, we have

$$
\mathcal{E}_{h}^{\frac{1}{2}}(t) \leqslant t \widetilde{K} h^{\theta}\left(\left\|w_{0}\right\|_{\frac{3}{2}}+\left\|w_{1}\right\|_{1}\right) \mathrm{d} t \quad(t \geqslant 0) .
$$

On the other hand, using (3.4), combined with (1.4) and (1.5), we have that there exists a constant $\widehat{h}^{*}>0$ such that, for every $h \in\left(0, \widehat{h}^{*}\right)$,

$$
\begin{aligned}
\left\|\dot{w}(t)-\dot{w}_{h}(t)\right\| \leqslant\left\|\dot{w}(t)-\pi_{h} \dot{w}(t)\right\|+\| \pi_{h} \dot{w}(t)- & \dot{w}_{h}(t) \| \\
& \leqslant K\left[h^{\theta}\left(\left\|w_{0}\right\|_{\frac{3}{2}}+\left\|w_{1}\right\|_{1}\right)+\mathcal{E}_{h}^{\frac{1}{2}}(t)\right], \\
\left\|w(t)-w_{h}(t)\right\|_{\frac{1}{2}} \leqslant\left\|w(t)-\pi_{h} w(t)\right\|_{\frac{1}{2}}+\| \pi_{h} w(t) & -w_{h}(t) \|_{\frac{1}{2}} \\
& \leqslant K\left[h^{\theta}\left(\left\|w_{0}\right\|_{\frac{3}{2}}+\left\|w_{1}\right\|_{1}\right)+\mathcal{E}_{h}^{\frac{1}{2}}(t)\right],
\end{aligned}
$$

for some constant $K>0$. The last two inequalities, combined to (3.6) yield the conclusion (3.3). 
For $h>0$ we denote $X_{h}=V_{h} \times V_{h}$ and we consider the operators

$$
\mathcal{A}_{h}=\left[\begin{array}{cc}
0 & I \\
-A_{0 h} & 0
\end{array}\right], \quad \mathcal{B}_{h}=\left[\begin{array}{c}
0 \\
B_{0 h}
\end{array}\right]
$$

The discrete analogues of the semigroups $\mathbb{T}, \mathbb{S}$ and of the operator $L_{t}$, denoted by $\mathbb{T}_{h}, \mathbb{S}_{h}$ and $L_{h, t}$ respectively, are defined, for every $h>0$, by

$$
\mathbb{T}_{h, t}=\mathrm{e}^{t\left(\mathcal{A}_{h}-\mathcal{B}_{h} \mathcal{B}_{h}^{*}\right)}, \quad \mathbb{S}_{h, t}=\mathrm{e}^{t\left(-\mathcal{A}_{h}-\mathcal{B}_{h} \mathcal{B}_{h}^{*}\right)}, \quad L_{h, t}=\mathbb{S}_{h, t} \mathbb{T}_{h, t} \quad(t \geqslant 0) .
$$

For every $h>0$ we define $\Pi_{h} \in \mathcal{L}\left(H_{\frac{1}{2}} \times H_{\frac{1}{2}}, X_{h}\right)$ by

$$
\Pi_{h}=\left[\begin{array}{cc}
\pi_{h} & 0 \\
0 & \pi_{h}
\end{array}\right]
$$

The following two results are consequences of Proposition 3.1.

Corollary 3.2. There exist two constants $C_{1}, h^{*}>0$ such that, for every $h \in\left(0, h^{*}\right)$ and $t>0$, we have (recall that $L_{t}=\mathbb{S}_{t} \mathbb{T}_{t}$ for every $t \geqslant 0$ ):

$$
\begin{array}{rlrl}
\left\|\Pi_{h} \mathbb{T}_{t} Z_{0}-\mathbb{T}_{h, t} \Pi_{h} Z_{0}\right\|_{X} \leqslant C_{1} t h^{\theta}\left\|Z_{0}\right\|_{H_{\frac{3}{2}} \times H_{1}} & & \left(Z_{0} \in H_{\frac{3}{2}} \times H_{1}\right), \\
\left\|\Pi_{h} \mathbb{S}_{t} Z_{0}-\mathbb{S}_{h, t} \Pi_{h} Z_{0}\right\|_{X} \leqslant C_{1} t h^{\theta}\left\|Z_{0}\right\|_{H_{\frac{3}{2}}} \times H_{1} & \left(Z_{0} \in H_{\frac{3}{2}} \times H_{1}\right), \\
\left\|\Pi_{h} L_{t} Z_{0}-L_{h, t} \Pi_{h} Z_{0}\right\|_{X} \leqslant C_{1} t h^{\theta}\left\|Z_{0}\right\|_{H_{\frac{3}{2}} \times H_{1}} & \left(Z_{0} \in H_{\frac{3}{2}} \times H_{1}\right) .
\end{array}
$$

Proof. The estimate (3.10) is nothing else but (3.6) rewritten in semigroup terms. To prove (3.11), it suffices to notice that $P \mathbb{S}_{t}=\mathbb{T}_{t} P$ where

$$
P\left[\begin{array}{l}
w_{0} \\
w_{1}
\end{array}\right]=\left[\begin{array}{c}
w_{0} \\
-w_{1}
\end{array}\right] \quad\left(\left[\begin{array}{l}
w_{0} \\
w_{1}
\end{array}\right] \in X\right)
$$

Finally, estimate (3.12) can be easily obtained from (3.10) and (3.11).

Corollary 3.3. There exist three constants $C_{0}, C_{1}, h^{*}>0$ such that, for every $t>0$, $h \in\left(0, h^{*}\right)$ and $k \in \mathbb{N}$ we have

$$
\left\|L_{t}^{k} Z_{0}-L_{h, t}^{k} \Pi_{h} Z_{0}\right\|_{X} \leqslant\left(C_{0}+k C_{1} t\right) h^{\theta}\left\|Z_{0}\right\|_{H_{\frac{3}{2}}} \times H_{1} \quad\left(Z_{0} \in H_{\frac{3}{2}} \times H_{1}\right) .
$$

Proof. We have

$$
\left\|L_{t}^{k} Z_{0}-L_{h, t}^{k} \Pi_{h} Z_{0}\right\|_{X} \leqslant\left\|L_{t}^{k} Z_{0}-\Pi_{h} L_{t}^{k} Z_{0}\right\|_{X}+\left\|\Pi_{h} L_{t}^{k} Z_{0}-L_{h, t}^{k} \Pi_{h} Z_{0}\right\|_{X} .
$$

From Proposition 2.5 it follows that, for every $t \geqslant 0, H_{\frac{3}{2}} \times H_{1}$ is an invariant space for $L_{t}$. Using this fact combined to (1.4) and (1.5) we obtain that there exists a constant $C_{0}>0$ such that the first term of the right-hand side of the above inequality satisfies

$$
\left\|L_{t}^{k} Z_{0}-\Pi_{h} L_{t}^{k} Z_{0}\right\|_{X} \leqslant C_{0} h^{\theta}\left\|Z_{0}\right\|_{H_{\frac{3}{2}}} \times H_{1} .
$$

For the second term of the right-hand side in (3.13) we have

$$
\begin{array}{r}
\left\|\Pi_{h} L_{t}^{k} Z_{0}-L_{h, t}^{k} \Pi_{h} Z_{0}\right\|_{X} \leqslant\left\|\Pi_{h} L_{t}^{k} Z_{0}-L_{h, t} \Pi_{h} L_{t}^{k-1} Z_{0}\right\|_{X}+\left\|L_{h, t} \Pi_{h} L_{t}^{k-1} Z_{0}-L_{h, t}^{k} \Pi_{h} Z_{0}\right\|_{X} \\
=\left\|\Pi_{h} L_{t}\left(L_{t}^{k-1} Z_{0}\right)-L_{h, t} \Pi_{h} L_{t}^{k-1} Z_{0}\right\|_{X}+\left\|L_{h, t}\left(\Pi_{h} L_{t}^{k-1} Z_{0}-L_{h, t}^{k-1} \Pi_{h} Z_{0}\right)\right\|_{X} .
\end{array}
$$


Applying (3.12), we obtain

$$
\left\|\Pi_{h} L_{t}^{k} Z_{0}-L_{h, t}^{k} \Pi_{h} Z_{0}\right\|_{X} \leqslant C_{1} t h^{\theta}\left\|Z_{0}\right\|_{H_{\frac{3}{2}} \times H_{1}}+\left\|\Pi_{h} L_{t}^{k-1} Z_{0}-L_{h, t}^{k-1} \Pi_{h} Z_{0}\right\|_{X} .
$$

By an obvious induction argument it follows that

$$
\left\|\Pi_{h} L_{t}^{k} Z_{0}-L_{h, t}^{k} \Pi_{h} Z_{0}\right\|_{X} \leqslant C_{1} t k h^{\theta}\left\|Z_{0}\right\|_{H_{\frac{3}{2}}} \times H_{1} \quad\left(Z_{0} \in H_{\frac{3}{2}} \times H_{1}\right) .
$$

Finally, combining (3.13)-(3.15), we obtain the conclusion of the corollary.

\section{Proof of the main result}

In this section we continue to use the notation from (3.7)-(3.9) for $\mathcal{A}_{h}, \mathcal{B}_{h}, \mathbb{T}_{h}, \mathbb{S}_{h}, L_{h}$ and $\Pi_{h}$. We first give the following result:

Lemma 4.1. Suppose that the system (1.2), (1.3) is exactly controllable in time $\tau>0$ and that $B_{0} B_{0}^{*} \in \mathcal{L}\left(H_{1}, H_{\frac{1}{2}}\right)$. Let $Q_{0}=\left[\begin{array}{l}q_{0} \\ q_{1}\end{array}\right] \in H_{\frac{3}{2}} \times H_{1}$ and let $u$ be the control given by (2.11), where $W_{0}=\left[\begin{array}{l}w_{0} \\ w_{1}\end{array}\right]$ is given by (2.10). Let $v_{h}:[0, \tau] \rightarrow U_{h}$ be defined by

$$
v_{h}(t)=\mathcal{B}_{h}^{*} \mathbb{T}_{h, t} \Pi_{h} W_{0}+\mathcal{B}_{h}^{*} \mathbb{S}_{h, \tau-t} \mathbb{T}_{h, \tau} \Pi_{h} W_{0} \quad(t \in[0, \tau])
$$

Then there exist three constants $C_{2}, C_{3}, h^{*}>0$ such that, for every $h \in\left(0, h^{*}\right)$, we have

$$
\left\|\left(u-v_{h}\right)(t)\right\|_{U} \leqslant \frac{C_{2}+t C_{3}}{1-\left\|L_{\tau}\right\|_{\mathcal{L}\left(H_{\frac{3}{2}} \times H_{1}\right)}} h^{\theta}\left\|Q_{0}\right\|_{H_{\frac{3}{2}} \times H_{1}} \quad(t \in[0, \tau]) .
$$

Proof. We first note that from Proposition 2.5 and the fact that $Q_{0} \in H_{\frac{3}{2}} \times H_{1}$ it follows that $W_{0}$ given by (2.10) still belongs to $H_{\frac{3}{2}} \times H_{1}$. Using (2.11), (4.1), (1.9) and (3.7) we see that for every $t \in[0, \tau]$ we have :

$$
\begin{array}{r}
\left\|\left(u-v_{h}\right)(t)\right\|_{U}=\left\|\mathcal{B}^{*} \mathbb{T}_{t} W_{0}+\mathcal{B}^{*} \mathbb{S}_{\tau-t} \mathbb{T}_{\tau} W_{0}-\mathcal{B}_{h}^{*} \mathbb{T}_{h, t} \Pi_{h} W_{0}-\mathcal{B}_{h}^{*} \mathbb{S}_{h, \tau-t} \mathbb{T}_{h, \tau} \Pi_{h} W_{0}\right\|_{U} \\
\leqslant\left\|\mathcal{B}^{*} \mathbb{T}_{t} W_{0}-\left[\begin{array}{ll}
0 & B_{0 h}^{*}
\end{array}\right] \mathbb{T}_{t} W_{0}\right\|_{U}+\left\|\left[\begin{array}{ll}
0 & B_{0 h}^{*}
\end{array}\right]\left(\mathbb{T}_{t} W_{0}-\mathbb{T}_{h, t} \Pi_{h} W_{0}\right)\right\|_{U} \\
+\left\|\mathcal{B}^{*} \mathbb{S}_{\tau-t} \mathbb{T}_{\tau} W_{0}-\left[\begin{array}{ll}
0 & B_{0 h}^{*}
\end{array}\right] \mathbb{S}_{\tau-t} \mathbb{T}_{\tau} W_{0}\right\|_{U} \\
+\left\|\left[\begin{array}{ll}
0 & B_{0 h}^{*}
\end{array}\right]\left(\mathbb{S}_{\tau-t} \mathbb{T}_{\tau} W_{0}-\mathbb{S}_{h, \tau-t} \mathbb{T}_{h, \tau} \Pi_{h} W_{0}\right)\right\|_{U}
\end{array}
$$

Let $h^{*}>0$ be chosen like in Proposition 3.1. To bound the first term in the right hand side of (4.3) we note that since $\mathcal{B}^{*}=\left[\begin{array}{ll}0 & B_{0}^{*}\end{array}\right]$ and $B_{0 h}^{*}=B_{0}^{*} \widetilde{\pi}_{h}$ we have that

$$
\left\|\mathcal{B}^{*} \mathbb{T}_{t} W_{0}-\left[\begin{array}{ll}
0 & B_{0 h}^{*}
\end{array}\right] \mathbb{T}_{t} W_{0}\right\|_{U}=\left\|B_{0}^{*}\left(\dot{w}(t)-\tilde{\pi}_{h} \dot{w}(t)\right)\right\|_{U} \leqslant\left\|B_{0}^{*}\right\|_{\mathcal{L}(H, U)}\left\|\dot{w}(t)-\tilde{\pi}_{h} \dot{w}(t)\right\|,
$$

where we have denoted $\mathbb{T}_{t} W_{0}=\left[\begin{array}{c}w(t) \\ \dot{w}(t)\end{array}\right]$. Using next (1.8) and Proposition 2.5 we obtain that there exists a constant $C_{0}>0$ such that

$$
\left\|\mathcal{B}^{*} \mathbb{T}_{t} W_{0}-\left[\begin{array}{ll}
0 & B_{0 h}^{*}
\end{array}\right] \mathbb{T}_{t} W_{0}\right\|_{U} \leqslant C_{0} h^{\theta}\|\dot{w}(t)\|_{\frac{1}{2}} \leqslant C_{0} h^{\theta}\left\|W_{0}\right\|_{H_{\frac{3}{2}}} \times H_{1} .
$$

Similarly we show that the third term in the right hand side of (4.3) satisfies

$$
\left\|\mathcal{B}^{*} \mathbb{S}_{\tau-t} \mathbb{T}_{\tau} W_{0}-\left[\begin{array}{ll}
0 & B_{0 h}^{*}
\end{array}\right] \mathbb{S}_{\tau-t} \mathbb{T}_{\tau} W_{0}\right\|_{U} \leqslant C_{0} h^{\theta}\left\|W_{0}\right\|_{H_{\frac{3}{2}}} \times H_{1}
$$


To bound the second term in the right hand side of (4.3) we use the uniform boundedness of the family of operators $\left(B_{0 h}^{*}\right)_{h \in\left(0, h^{*}\right)}$ in $\mathcal{L}(H, U)$ and Proposition 3.1 to get

$$
\left\|\left[0 \quad B_{0 h}^{*}\right]\left(\mathbb{T}_{t} W_{0}-\mathbb{T}_{h, t} \Pi_{h} W_{0}\right)\right\|_{U} \leqslant\left(K_{0}+K_{1} t\right) h^{\theta}\left\|W_{0}\right\|_{H_{\frac{3}{2}}} \times H_{1} .
$$

The forth term in the right hand side of (4.3) can be estimated similarly to get

$$
\left\|\left[\begin{array}{ll}
0 & B_{0 h}^{*}
\end{array}\right]\left(\mathbb{S}_{\tau-t} \mathbb{T}_{\tau} W_{0}-\mathbb{S}_{h, \tau-t} \mathbb{T}_{h, \tau} \Pi_{h} W_{0}\right)\right\|_{U} \leqslant\left(K_{0}+K_{1} t\right) h^{\theta}\left\|W_{0}\right\|_{H_{\frac{3}{2}}} \times H_{1}
$$

Using (4.4)-(4.7), relation (4.3) yields

$$
\left\|\left(u-v_{h}\right)(t)\right\|_{U} \leqslant\left(C_{2}+t C_{3}\right) h^{\theta}\left\|W_{0}\right\|_{H_{\frac{3}{2}}} \times H_{1},
$$

for some constants $C_{2}, C_{3}>0$ and $h \in\left(0, h^{*}\right)$. Using in the above estimate the fact, following from Proposition 2.5 and (2.10), that

$$
\left\|W_{0}\right\|_{H_{\frac{3}{2}} \times H_{1}} \leqslant \frac{1}{1-\left\|L_{\tau}\right\|_{\mathcal{L}\left(H_{\frac{3}{2}} \times H_{1}\right)}}\left\|Q_{0}\right\|_{H_{\frac{3}{2}} \times H_{1}},
$$

we obtain the conclusion of this lemma.

We are now in a position to prove the main result of this work.

Proof of Theorem 1.1. Using the semigroup notation introduced in Section 2 we can write $u_{h}$ given by (1.16) as

$$
u_{h}(t)=\mathcal{B}_{h}^{*} \mathbb{T}_{h, t}\left[\begin{array}{c}
w_{0 h} \\
w_{1 h}
\end{array}\right]+\mathcal{B}_{h}^{*} \mathbb{S}_{h, \tau-t} \mathbb{T}_{h, \tau}\left[\begin{array}{l}
w_{0 h} \\
w_{1 h}
\end{array}\right] \quad(t \in[0, \tau]),
$$

where

$$
\left[\begin{array}{l}
w_{0 h} \\
w_{1 h}
\end{array}\right]=\sum_{n=0}^{N(h)} L_{h, \tau}^{n} \Pi_{h}\left[\begin{array}{l}
q_{0} \\
q_{1}
\end{array}\right]
$$

Since

$$
\left\|u-u_{h}\right\|_{L^{2}([0, \tau], U)} \leqslant\left\|u-v_{h}\right\|_{L^{2}([0, \tau], U)}+\left\|v_{h}-u_{h}\right\|_{L^{2}([0, \tau], U)}
$$

it suffices to evaluate the two terms from the right, where $v_{h}$ is given by (4.1).

To estimate the second term in the right-hand side of (4.10) we first note that

$$
\begin{aligned}
& \left(v_{h}-u_{h}\right)(t) \\
& \quad=\mathcal{B}_{h}^{*} \mathbb{T}_{h, t} \Pi_{h} W_{0}+\mathcal{B}_{h}^{*} \mathbb{S}_{h, \tau-t} \mathbb{T}_{h, \tau} \Pi_{h} W_{0}-\mathcal{B}_{h}^{*} \mathbb{T}_{h, t} \Pi_{h}\left[\begin{array}{c}
w_{0 h} \\
w_{1 h}
\end{array}\right]-\mathcal{B}_{h}^{*} \mathbb{S}_{h, \tau-t} \mathbb{T}_{h, \tau} \Pi_{h}\left[\begin{array}{c}
w_{0 h} \\
w_{1 h}
\end{array}\right] .
\end{aligned}
$$

It follows that there exists a positive constant $C$ with

$$
\begin{aligned}
&\left\|\left(v_{h}-u_{h}\right)(t)\right\|_{U} \leqslant\left\|\mathcal{B}_{h}^{*} \mathbb{T}_{h, t} \Pi_{h} W_{0}-\mathcal{B}_{h}^{*} \mathbb{T}_{h, t} \Pi_{h}\left[\begin{array}{c}
w_{0 h} \\
w_{1 h}
\end{array}\right]\right\|_{U} \\
&+\left\|\mathcal{B}_{h}^{*} \mathbb{S}_{h, \tau-t} \mathbb{T}_{h, \tau} \Pi_{h} W_{0}-\mathcal{B}_{h}^{*} \mathbb{S}_{h, \tau-t} \mathbb{T}_{h, \tau} \Pi_{h}\left[\begin{array}{c}
w_{0 h} \\
w_{1 h}
\end{array}\right]\right\|_{U} \\
& \leqslant C\left\|W_{0}-\left[\begin{array}{c}
w_{0 h} \\
w_{1 h}
\end{array}\right]\right\|_{X}=C\left\|\sum_{n=0}^{\infty} L_{\tau}^{n} Q_{0}-\sum_{n=0}^{N(h)} L_{h, \tau}^{n} \Pi_{h} Q_{0}\right\|_{X} \\
& \leqslant C \sum_{n=N(h)+1}^{\infty}\left\|L_{\tau}\right\|_{\mathcal{L}(X)}^{n}\left\|Q_{0}\right\|_{X}+C \sum_{n=0}^{N(h)}\left\|\left(L_{\tau}^{n}-L_{h, \tau}^{n} \Pi_{h}\right) Q_{0}\right\|_{X} .
\end{aligned}
$$


The above estimate and Corollary 3.3 imply that there exists $\widetilde{C}>0$ such that

$$
\begin{aligned}
\left\|\left(v_{h}-u_{h}\right)(t)\right\|_{U} & \leqslant C \frac{\left\|L_{\tau}\right\|_{\mathcal{L}(X)}^{N(h)+1}}{1-\left\|L_{\tau}\right\|_{\mathcal{L}(X)}}\left\|Q_{0}\right\|_{X}+C h^{\theta} \sum_{n=0}^{N}\left(C_{0}+n C_{1} \tau\right)\left\|Q_{0}\right\|_{H_{\frac{3}{2}}} \times H_{1} \\
& =C \frac{\left\|L_{\tau}\right\|_{\mathcal{L}(X)}^{N(h)+1}}{1-\left\|L_{\tau}\right\|_{\mathcal{L}(X)}}\left\|Q_{0}\right\|_{X}+\widetilde{C} N^{2}(h)(1+\tau) h^{\theta}\left\|Q_{0}\right\|_{H_{\frac{3}{2}}} \times H_{1} \\
& \leqslant \frac{\widetilde{C}(1+\tau)}{1-\left\|L_{\tau}\right\|_{\mathcal{L}(X)}}\left(\left\|L_{\tau}\right\|_{\mathcal{L}(X)}^{N(h)}+N^{2}(h) h^{\theta}\right)\left\|Q_{0}\right\|_{H_{\frac{3}{2}}} \times H_{1}
\end{aligned}
$$

By choosing $N(h)=\left[\frac{\theta}{\ln \left(\left\|L_{\tau}\right\|_{\mathcal{L}(X)}\right)} \ln (h)\right]$ we deduce that

$$
\left\|\left(v_{h}-u_{h}\right)(t)\right\|_{U} \leqslant \frac{\widetilde{C}(1+\tau)}{\left(1-\left\|L_{\tau}\right\|_{\mathcal{L}(X)}\right) \ln ^{2}\left(\left\|L_{\tau}\right\|_{\mathcal{L}(X)}^{-1}\right)} \ln ^{2}\left(h^{-1}\right) h^{\theta}\left\|Q_{0}\right\|_{H_{\frac{3}{2}}} \times H_{1} .
$$

Combining this last estimate with (4.2) and taking $m_{\tau}=\frac{1}{\ln \left(\left\|L_{\tau}\right\|_{\mathcal{L}(X)}^{-1}\right)}$ we obtain the conclusion (1.21).

Remark 4.2. The functions $u_{h}$ given by (1.16) or $v_{h}$ from (4.1) should not be confused with the exact control $\zeta_{h}$, obtained by applying Russell's principle to the finite-dimensional system

$$
\begin{aligned}
& \ddot{q}_{h}(t)+A_{0 h} q_{h}(t)+B_{0 h} u=0, \\
& q_{h}(0)=\pi_{h} q_{0}, \quad \dot{q}_{h}(0)=\pi_{h} q_{1} .
\end{aligned}
$$

Indeed, this control $\zeta_{h}$ is given by the formula

$$
\zeta_{h}(t)=\mathcal{B}_{h}^{*} \mathbb{T}_{h, t} Z_{h 0}+\mathcal{B}_{h}^{*} \mathbb{S}_{h, \tau-t} \mathbb{T}_{h, \tau} Z_{h 0}, \quad Z_{h 0}=\left(I-L_{h, \tau}\right)^{-1} \Pi_{h}\left[\begin{array}{c}
q_{0} \\
q_{1}
\end{array}\right],
$$

so that $u_{h}$ is obtained by "filtering" (in an appropriate sense) $\zeta_{h}$. Note that, since $\mathbb{T}_{h}$ and $\mathbb{S}_{h}$ are not, in general, uniformly exponentially stable (with respect to $h$ ), the control $\zeta_{h}$ does not, in general, converge to u (see, for instance, [21]).

\section{$5 \quad$ Examples and numerical results}

In this section we apply our numerical method to approximate exact controls for the two dimensional wave equation and for the Euler-Bernoulli beam equation. For both examples we consider distributed controls.

\subsection{The wave equation}

In this subsection we consider the approximation of an internal distributed exact control for the wave equation with homogeneous Dirichlet boundary condition.

Let $\Omega \subset \mathbb{R}^{2}$ be an open connected set with boundary of class $C^{2}$ or let $\Omega$ be a rectangular domain. Let $\mathcal{O} \subset \Omega, \mathcal{O} \neq \Omega$ be an open set. We consider the control problem

$$
\ddot{q}(x, t)-\Delta q(x, t)+\chi_{\mathcal{O}}(x) u(x, t)=0, \quad(x, t) \in \Omega \times[0, \tau]
$$




$$
\begin{gathered}
q(x, t)=0, \quad(x, t) \in \partial \Omega \times[0, \tau] \\
q(x, 0)=q_{0}(x), \quad \dot{q}(x, 0)=q_{1}(x), \quad x \in \Omega,
\end{gathered}
$$

where $\chi_{\mathcal{O}} \in \mathcal{D}(\Omega)$ is such that $\chi_{\mathcal{O}}(x)=1$ for $x \in \mathcal{O}$ and $\chi_{\mathcal{O}}(x) \geqslant 0$ for $x \in \Omega$.

In order to apply the method described in (1.11)-(1.20) to this case we need appropriate choices of spaces and operators. We take $H=L^{2}(\Omega), U=H$ and $A_{0}: \mathcal{D}\left(A_{0}\right) \rightarrow H$ with

$$
\mathcal{D}\left(A_{0}\right)=\mathcal{H}^{2}(\Omega) \cap \mathcal{H}_{0}^{1}(\Omega), \quad A_{0} \varphi=-\Delta \varphi \quad\left(\varphi \in \mathcal{D}\left(A_{0}\right)\right),
$$

where we use the notation $\mathcal{H}^{m}(\Omega)$, with $m \in \mathbb{N}$, for the standard Sobolev spaces. It is well known that $A_{0}$ is a self-adjoint, strictly positive operator with compact resolvents. The corresponding spaces $H_{\frac{3}{2}}, H_{1}$ and $H_{\frac{1}{2}}$ introduced in Section 1 are in this case given by

$$
\begin{gathered}
H_{\frac{3}{2}}=\left\{\varphi \in \mathcal{H}^{3}(\Omega) \cap \mathcal{H}_{0}^{1}(\Omega) \mid \Delta \varphi=0 \text { on } \partial \Omega\right\}, \\
H_{1}=\mathcal{H}^{2}(\Omega) \cap \mathcal{H}_{0}^{1}(\Omega), \quad H_{\frac{1}{2}}=\mathcal{H}_{0}^{1}(\Omega) .
\end{gathered}
$$

The control operator $B_{0} \in \mathcal{L}(H)$ is defined by

$$
B_{0} u=\chi_{\mathcal{O}} u \quad(u \in H) .
$$

The operator $B_{0}$ is clearly self-adjoint and $B_{0} B_{0}^{*} \in \mathcal{L}\left(H_{1}, H_{\frac{1}{2}}\right)$. Moreover, we assume that $\tau$ and $\mathcal{O}$ are such that the system (5.1)-(5.3) is exactly controllable in time $\tau$, i.e., that for every $\left[\begin{array}{l}q_{0} \\ q_{1}\end{array}\right] \in H_{0}^{1}(\Omega) \times L^{2}(\Omega)$ there exists a control $u \in L^{2}([0, \tau], U)$ such that $q(\tau)=\dot{q}(\tau)=0$. Sufficient conditions in which this assumption holds are give in various works, see Lions [11], Bardos, Lebeau and Rauch [2], Liu [12].

To construct an approximating family of spaces $\left(V_{h}\right)_{h>0}$ we consider a quasi-uniform triangulation $\mathcal{T}_{h}$ of $\Omega$ of diameter $h$, as defined, for instance, in [3, p.106]. For each $h>0$ we define $V_{h}$ by

$$
V_{h}=\left\{\varphi \in C(\bar{\Omega}) \mid \varphi_{\mid T} \in P_{1}(T) \text { for every } T \in \mathcal{T}_{h}, \varphi_{\mid \partial \Omega}=0\right\},
$$

where $P_{1}(T)$ is the set of affine functions on $T$. It is well-known (see, for instance, [13, p.96-97]) that the orthogonal projector $\pi_{h}$ from $H_{\frac{1}{2}}=\mathcal{H}_{0}^{1}(\Omega)$ onto $V_{h}$ satisfies (1.4) and (1.5) for $\theta=1$.

We define $U_{h}=\left\{\chi_{\mathcal{O}} v_{h} \mid v_{h} \in V_{h}\right\} \subset U$ and let $B_{0 h} \in \mathcal{L}(H)$ be given by $B_{0 h} \varphi=\widetilde{\pi}_{h}\left(\chi_{\mathcal{O}} \varphi\right)$ for every $\varphi \in H$. Note that $B_{0 h}^{*} \varphi_{h}=\chi_{\mathcal{O}} \varphi_{h}$ and $\left\langle B_{0 h} B_{0 h}^{*} \varphi_{h}, \psi_{h}\right\rangle=\left\langle\chi_{\mathcal{O}}^{2} \varphi_{h}, \psi_{h}\right\rangle$ for every $\varphi_{h}, \psi_{h} \in V_{h}$, where $\langle\cdot, \cdot\rangle$ denotes the inner product in $L^{2}(\Omega)$.

With the above choice of spaces and operators, denoting by $N(h)=\left[\frac{\theta}{\ln \left\|L_{\tau}\right\|} \ln h\right]$, the first part of the general method described in (1.11)-(1.20) reduces to the computation of the families of functions $\left(w_{h}^{n}\right)_{1 \leqslant n \leqslant N(h)},\left(w_{b, h}^{n}\right)_{1 \leqslant n \leqslant N(h)}$ satisfying, for every $v_{h} \in V_{h}$,

$$
\begin{gathered}
\left\langle\ddot{w}_{h}^{n}(t), v_{h}\right\rangle+\left\langle\nabla w_{h}^{n}(t), \nabla v_{h}\right\rangle+\left\langle\chi_{\mathcal{O}}^{2} \dot{w}_{h}^{n}(t), v_{h}\right\rangle=0 \quad(t \in[0, \tau]) \\
w_{h}^{n}(0)=\left\{\begin{array}{ll}
\pi_{h} q_{0}, & \text { if } n=1 \\
w_{b, h}^{n-1}(0), & \text { if } n>1
\end{array} \quad \dot{w}_{h}^{n}(0)= \begin{cases}\pi_{h} q_{1}, & \text { if } n=1 \\
\dot{w}_{b, h}^{n-1}(0), & \text { if } n>1,\end{cases} \right.
\end{gathered}
$$

and

$$
\left\langle\ddot{w}_{b, h}^{n}(t), v_{h}\right\rangle+\left\langle\nabla w_{b, h}^{n}(t), \nabla v_{h}\right\rangle-\left\langle\chi_{\mathcal{O}}^{2} \dot{w}_{b, h}^{n}(t), v_{h}\right\rangle=0 \quad(t \in[0, \tau])
$$




$$
w_{b, h}^{n}(\tau)=w_{h}^{n}(\tau), \quad \dot{w}_{b, h}^{n}(\tau)=\dot{w}_{h}^{n}(\tau) .
$$

The second part of the method described in (1.11)-(1.20) reduces to the computation of $w_{0 h}$ and $w_{1 h}$ defined by

$$
\left[\begin{array}{l}
w_{0 h} \\
w_{1 h}
\end{array}\right]=\left[\begin{array}{l}
\pi_{h} q_{0} \\
\pi_{h} q_{1}
\end{array}\right]+\sum_{n=1}^{N(h)}\left[\begin{array}{l}
w_{b, h}^{n}(0) \\
\dot{w}_{b, h}^{n}(0)
\end{array}\right] .
$$

Finally, the approximation $u_{h}$ of the exact control $u$ is given by

$$
u_{h}=\chi_{\mathcal{O}} \dot{w}_{h}+\chi_{\mathcal{O}} \dot{w}_{b, h},
$$

where $w_{h}$ and $w_{b, h}$ are the solution of

$$
\begin{gathered}
\left\langle\ddot{w}_{h}(t), v_{h}\right\rangle+\left\langle\nabla w_{h}(t), \nabla v_{h}\right\rangle+\left\langle\chi_{\mathcal{O}}^{2} \dot{w}_{h}(t), v_{h}\right\rangle=0 \quad\left(v_{h} \in V_{h}, t \in[0, \tau]\right) \\
w_{h}(0)=w_{0 h}, \quad \dot{w}_{h}(0)=w_{1 h} \\
\left\langle\ddot{w}_{b, h}(t), v_{h}\right\rangle+\left\langle\nabla w_{b, h}(t), \nabla v_{h}\right\rangle-\left\langle\chi_{\mathcal{O}}^{2} \dot{w}_{b, h}(t), v_{h}\right\rangle=0 \quad\left(v_{h} \in V_{h}, t \in[0, \tau]\right) \\
w_{b, h}(\tau)=w_{h}(\tau), \quad \dot{w}_{b, h}(\tau)=\dot{w}_{h}(\tau) .
\end{gathered}
$$

Since we checked above all the necessary assumptions, we can apply Theorem 1.1 to obtain that $\left(u_{h}\right)$ converges in $C\left([0, \tau] ; L^{2}(\Omega)\right)$ to an exact control $u$ such that

$$
\left\|u-u_{h}\right\|_{C\left([0, \tau] ; L^{2}(\Omega)\right)} \leqslant C h \ln ^{2}\left(h^{-1}\right)\left(\left\|q_{0}\right\|_{\mathcal{H}^{3}(\Omega)}+\left\|q_{1}\right\|_{\mathcal{H}^{2}(\Omega)}\right) \quad\left(0<h<h^{*}\right) .
$$

for some constants $h^{*}, C>0$.

The efficiency of the algorithm has been tested in the case $\Omega=[0,1]^{2}$ and $\mathcal{O}=$ $\left[\left(x_{1}, x_{2}\right) \times(0,1)\right] \cup\left[(0,1) \times\left(y_{1}, y_{2}\right)\right]$, where $x_{1}, x_{2}, y_{1}, y_{2} \in(0,1)$ are such that $x_{1}<x_{2}$ and $y_{1}<y_{2}$. The initial data that we want to steer to zero are the "bubble" functions $q_{0}(x, y)=q_{1}(x, y)=x^{3} y^{3}(1-x)^{3}(1-y)^{3}$ and the control time is $\tau=2 \sqrt{2}$. Note that $\left[\begin{array}{c}q_{0} \\ q_{1}\end{array}\right] \in H_{\frac{3}{2}} \times H_{\frac{1}{2}}$. We use 60 points of discretization in each space direction. For the time discretization we used a classical centered-difference implicit scheme and the CFL number is $\alpha=1 / 20$.

Figure 1 shows the norm decay of the solution of the discretized wave equation coresponding to (5.1)-(5.3), with a control $u_{h}$ given by (1.16).

Figure 2 displays the norm of the solution of the controlled discretized wave equation, corresponding to (5.1)-(5.3), at the time $\tau$ for different values of $N$ used in calculus of $\left[\begin{array}{l}w_{0 h} \\ w_{1 h}\end{array}\right]$.

\subsection{The Euler-Bernoulli beam equation}

This subsection is dedicated to the problem of the approximation of an internal distributed exact control for the Euler-Bernoulli beam equation.

Let $\Omega=(0,1)$ and let $\mathcal{O} \subset \Omega$ be an open and nonempty interval included in $\Omega$. We consider the following problem

$$
\begin{array}{r}
\ddot{q}(x, t)+\frac{\partial^{4} q}{\partial x^{4}}(x, t)+\chi_{\mathcal{O}}(x) u(x, t)=0, \quad(x, t) \in \Omega \times[0, \tau] \\
q(0, t)=\frac{\partial^{2} q}{\partial x^{2}}(0, t)=q(1, t)=\frac{\partial^{2} q}{\partial x^{2}}(1, t)=0, \quad t \in[0, \tau] \\
q(x, 0)=q_{0}(x), \quad \dot{q}(x, 0)=q_{1}(x), \quad x \in \Omega,
\end{array}
$$




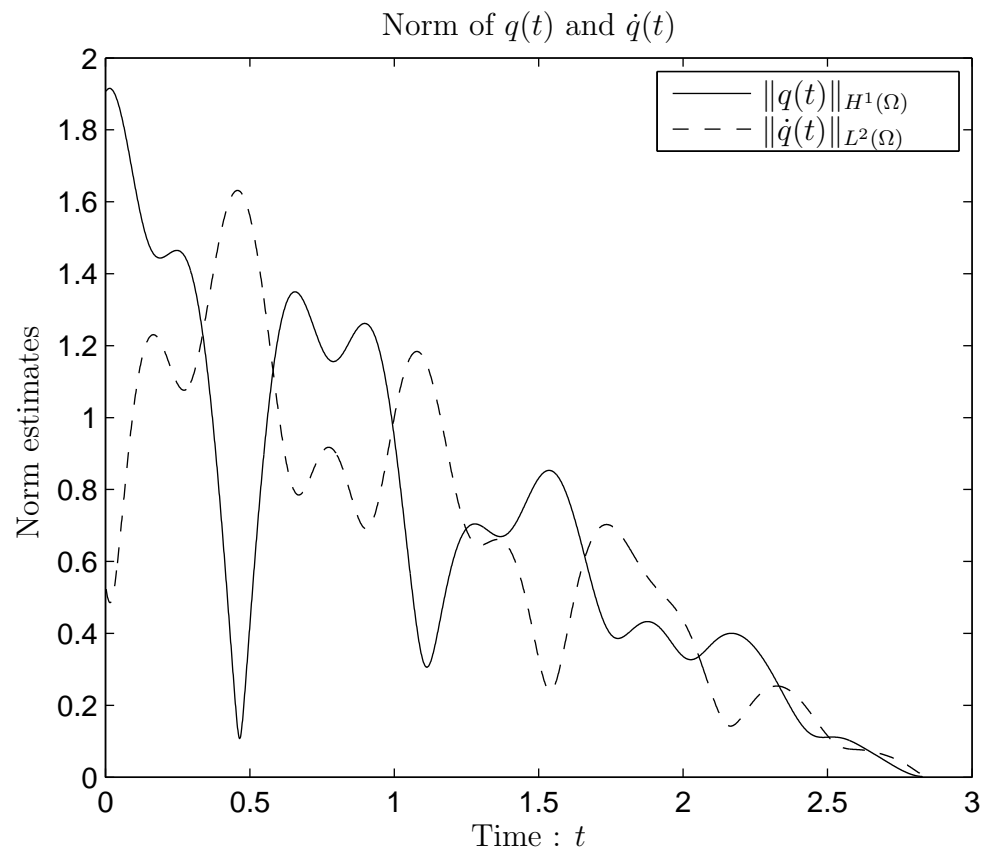

Figure 1: The norms of the solution of the controlled wave equation with the control $u_{h}$ given by (1.16). In continuous line is the norm $H_{0}^{1}$ of $q(t)$ and in dashed line the norm $L^{2}$ of $\dot{q}(t)$.

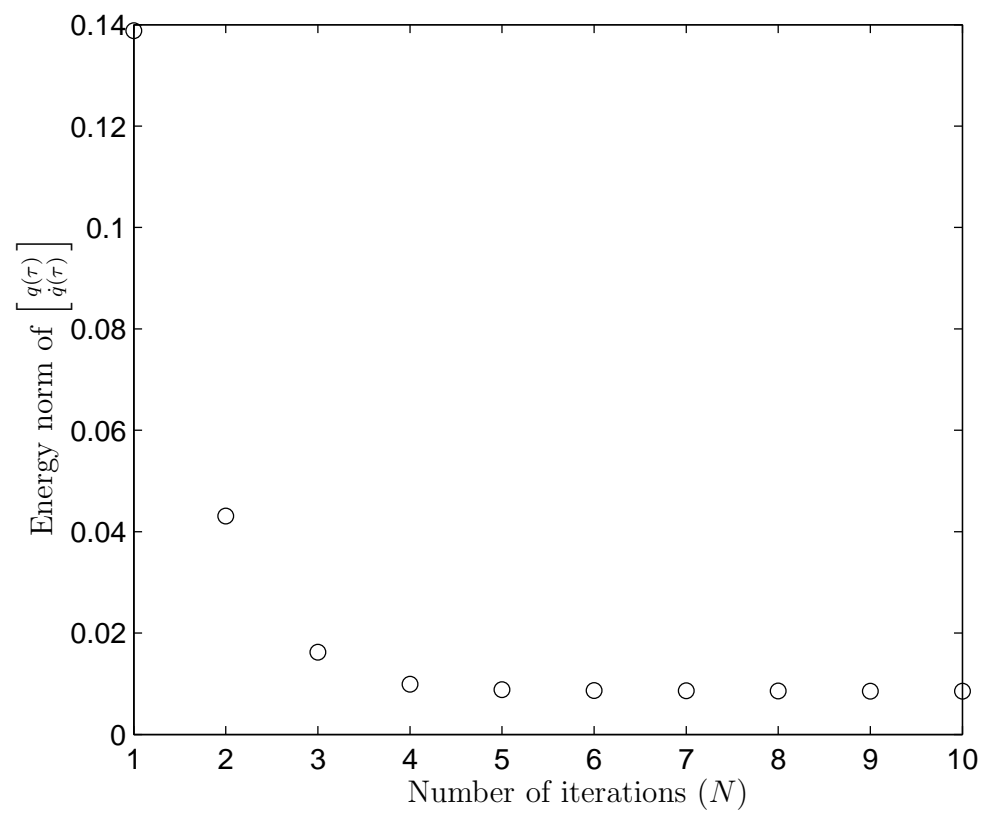

Figure 2: The energy of the controlled wave equation solution at the time $\tau$ versus the number of terms $N$ in the approximation of the control $u_{h}$.

modeling a beam hinged at the both ends with a control $u$ applied in an internal region. We denote by $\chi_{\mathcal{O}} \in \mathcal{D}(\Omega)$ a positive function which satisfies $\chi_{\mathcal{O}}(x)=1$ for every $x \in \mathcal{O}$. It is well known (see, for instance, [19, Example 6.8.3]) that the system (5.15)-(5.17) is exactly controllable in any time $\tau>0$. 
In order to apply the method described in this paper we need to choose appropriate spaces and operators. Let $H=L^{2}(\Omega), U=H$ and consider the operator $A_{0}: \mathcal{D}\left(A_{0}\right) \rightarrow H$, defined by

$$
\begin{gathered}
\mathcal{D}\left(A_{0}\right)=\left\{\varphi \in \mathcal{H}^{4}(\Omega) \mid \varphi(0)=\frac{d^{2} \varphi}{d x^{2}}(0)=\varphi(1)=\frac{d^{2} \varphi}{d x^{2}}(1)=0\right\}, \\
A_{0} \varphi=\frac{d^{4} \varphi}{d x^{4}} \quad\left(\varphi \in \mathcal{D}\left(A_{0}\right)\right) .
\end{gathered}
$$

It is well known that $A_{0}$ is a self-adjoint, strictly positive operator with compact resolvents. The corresponding spaces $H_{\frac{3}{2}}, H_{1}$ and $H_{\frac{1}{2}}$ introduced in Section 1 are now given by

$$
\begin{gathered}
H_{\frac{3}{2}}=\left\{\varphi \in \mathcal{H}^{6}(\Omega) \quad \mid \varphi(0)=\varphi(1)=\frac{d^{2} \varphi}{d x^{2}}(0)=\frac{d^{2} \varphi}{d x^{2}}(1)=\frac{d^{4} \varphi}{d x^{4}}(0)=\frac{d^{4} \varphi}{d x^{4}}(1)=0\right\}, \\
H_{1}=\left\{\varphi \in \mathcal{H}^{4}(\Omega) \mid \varphi(0)=\frac{d^{2} \varphi}{d x^{2}}(0)=\varphi(1)=\frac{d^{2} \varphi}{d x^{2}}(1)=0\right\}, \quad H_{\frac{1}{2}}=\mathcal{H}^{2}(\Omega) \cap \mathcal{H}_{0}^{1}(\Omega) .
\end{gathered}
$$

As in the case of the wave equation the control operator $B_{0} \in \mathcal{L}(H)$ is defined by $B_{0} u=$ $\chi_{\mathcal{O}} u$ for every $u \in H$. Clearly $B_{0}$ is self-adjoint and $B_{0} \in \mathcal{L}\left(H_{1}, H_{\frac{1}{2}}\right)$.

To construct an approximating family of spaces $\left(V_{h}\right)_{h>0}$ we consider an uniform discretization $\mathcal{I}_{h}$ of the interval $(0,1)$ formed by $\mathcal{N}$ points and $h=1 /(\mathcal{N}-1)$. For each $h>0$ we define $V_{h}$ by

$$
V_{h}=\left\{\varphi \in C^{1}([0,1]) \mid \varphi_{\mid I} \in P_{3}(T) \text { for every } I \in \mathcal{I}_{h}, \quad \varphi(0)=\varphi(1)=0\right\},
$$

where $P_{3}(I)$ is the set of polynomial functions of degree 3 on $I$. Note that $V_{h}$ is the cubic Hermite finite element space. Denoting by $\pi_{h}$ the orthogonal projector from $H_{\frac{1}{2}}$ to $V_{h}$ and applying the Theorem 3.3 from Strang and Fix [17, p. 144] we obtain estimates (1.4) and (1.5) with $\theta=2$.

The method described by (1.11)-(1.20) reduces to the computation of the families of functions $\left(w_{h}^{n}\right)_{1 \leqslant n \leqslant N(h)}, \quad\left(w_{b, h}^{n}\right)_{1 \leqslant n \leqslant N(h)}$ satisfying, for every $v_{h} \in V_{h}$,

$$
\begin{gathered}
\left\langle\ddot{w}_{h}^{n}(t), v_{h}\right\rangle+\left\langle\frac{\partial^{2} w_{h}^{n}}{\partial x^{2}}(t), \frac{\mathrm{d}^{2} v_{h}}{\mathrm{~d} x^{2}}\right\rangle+\left\langle\chi_{\mathcal{O}}^{2} \dot{w}_{h}^{n}(t), v_{h}\right\rangle=0 \\
w_{h}^{n}(0)=\left\{\begin{array}{ll}
\pi_{h} q_{0}, & \text { if } n=1 \\
w_{b, h}^{n-1}(0), & \text { if } n>1
\end{array} \quad \dot{w}_{h}^{n}(0)= \begin{cases}\pi_{h} q_{1}, & \text { if } n=1 \\
\dot{w}_{b, h}^{n-1}(0), & \text { if } n>1,\end{cases} \right.
\end{gathered}
$$

and

$$
\begin{gathered}
\left\langle\ddot{w}_{b, h}^{n}(t), v_{h}\right\rangle+\left\langle\frac{\partial^{2} w_{b, h}^{n}}{\partial x^{2}}(t), \frac{\mathrm{d}^{2} v_{h}}{\mathrm{~d} x^{2}}\right\rangle-\left\langle\chi_{\mathcal{O}}^{2} \dot{w}_{b, h}^{n}(t), v_{h}\right\rangle=0 \quad(t \in[0, \tau]) \\
w_{b, h}^{n}(\tau)=w_{h}^{n}(\tau), \quad \dot{w}_{b, h}^{n}(\tau)=\dot{w}_{h}^{n}(\tau) .
\end{gathered}
$$

The second part of the method described in (1.11)-(1.20) reduces to the computation of $w_{0 h}$ and $w_{1 h}$ defined by

$$
\left[\begin{array}{l}
w_{0 h} \\
w_{1 h}
\end{array}\right]=\left[\begin{array}{l}
\pi_{h} q_{0} \\
\pi_{h} q_{1}
\end{array}\right]+\sum_{n=1}^{N(h)}\left[\begin{array}{c}
w_{b, h}^{n}(0) \\
\dot{w}_{b, h}^{n}(0)
\end{array}\right] .
$$


Finally, the approximation $u_{h}$ of the exact control $u$ is given by

$$
u_{h}=\chi_{\mathcal{O}} \dot{w}_{h}+\chi_{\mathcal{O}} \dot{w}_{b, h},
$$

where $w_{h}$ and $w_{b, h}$ are the solution of

$$
\begin{gathered}
\left\langle\ddot{w}_{h}(t), v_{h}\right\rangle+\left\langle\frac{\partial^{2} w_{h}}{\partial x^{2}}(t), \frac{\mathrm{d}^{2} v_{h}}{\mathrm{~d} x^{2}}\right\rangle+\left\langle\chi_{\mathcal{O}}^{2} \dot{w}_{h}(t), v_{h}\right\rangle=0 \quad(t \in[0, \tau]) \\
w_{h}(0)=w_{0 h}, \quad \dot{w}_{h}(0)=w_{1 h} \\
\left\langle\ddot{w}_{b, h}(t), v_{h}\right\rangle+\left\langle\frac{\partial^{2} w_{b, h}}{\partial x^{2}}(t), \frac{\mathrm{d}^{2} v_{h}}{\mathrm{~d} x^{2}}\right\rangle-\left\langle\chi_{\mathcal{O}}^{2} \dot{w}_{b, h}(t), v_{h}\right\rangle=0 \quad(t \in[0, \tau]) \\
w_{b, h}(\tau)=w_{h}(\tau), \quad \dot{w}_{b, h}(\tau)=\dot{w}_{h}(\tau) .
\end{gathered}
$$

From Theorem 1.1 we obtain that $\left(u_{h}\right)$ converges in $C\left([0, \tau] ; L^{2}(\Omega)\right)$ to an exact control $u$ such that

$$
\left\|u-u_{h}\right\|_{C\left([0, \tau] ; L^{2}(\Omega)\right)} \leqslant C h^{2} \ln ^{2}\left(h^{-1}\right)\left(\left\|q_{0}\right\|_{\mathcal{H}^{6}(\Omega)}+\left\|q_{1}\right\|_{\mathcal{H}^{4}(\Omega)}\right) \quad\left(0<h<h^{*}\right) .
$$

for some constants $h^{*}, C>0$.

We tested the algorithm in the case $\mathcal{O}=\left(\frac{1}{3}, \frac{2}{3}\right)$ and the initial data that we want to steer to zero are $q_{0}(x)=x^{5}(1-x)^{5}, q_{1}(x)=-q_{0}(x)$ and the control time is $\tau=1$. Note that $\left[\begin{array}{l}q_{0} \\ q_{1}\end{array}\right] \in H_{\frac{3}{2}} \times H_{1}$. We used $\mathcal{N}=100$ discretization points in space and in time an implicit centered-difference scheme with the CFL number equal to 0.1.

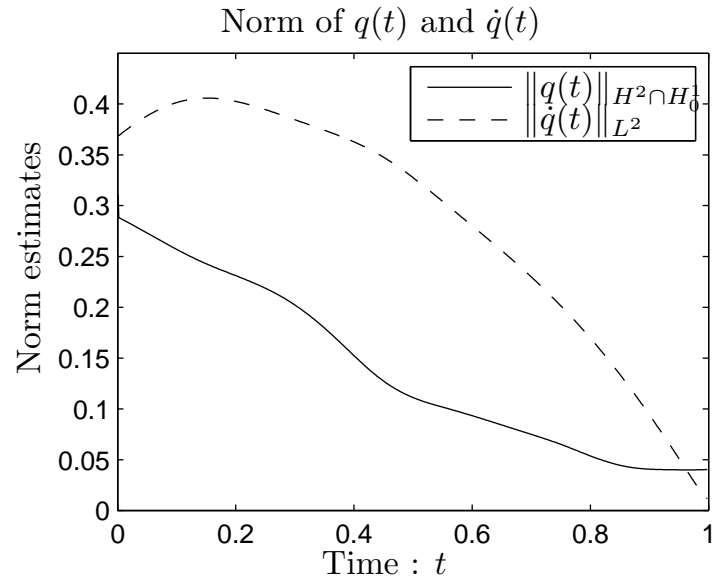

(a)

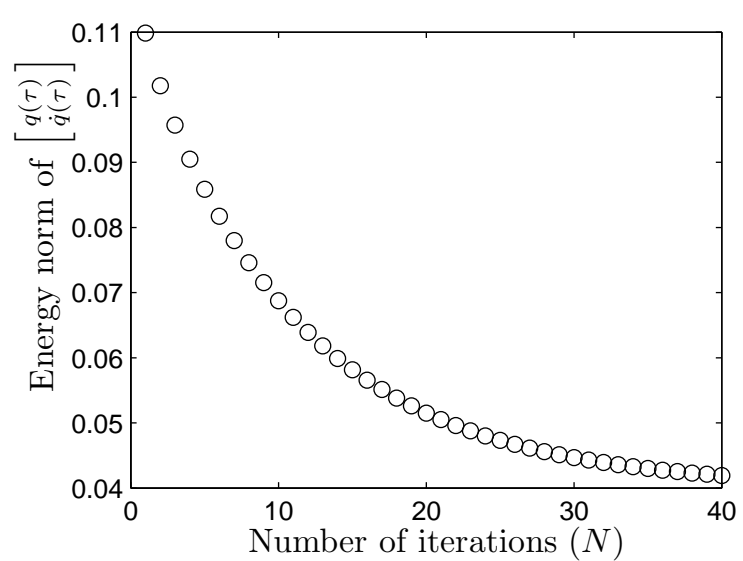

(b)

Figure 3: (a) The norm of the solution of the controlled beam equation, with $u=u_{h}$ and the initial state $q_{0}(x)=x^{5}(1-x)^{5}, q_{1}(x)=-q_{0}(x)$. (b) The energy of the solution of the controlled beam at time $\tau$ versus the number of terms $N$ in the approximation of $u_{h}$.

Figure 3(a) shows the norm decay of the solution of the discretized beam equation coresponding to (5.15)-(5.17), with a control $u_{h}$ given by (1.16). Figure 3(b) displays the dependence of the norm of the solution of (5.15)-(5.17), at the time $\tau$, to the number $N$ of terms used in the calculus of $\left[\begin{array}{l}w_{0 h} \\ w_{1 h}\end{array}\right]$.

In Figure 4 is given the form of the approximate control $u_{h}$ corresponding to the initial data $\left[\begin{array}{l}q_{0} \\ q_{1}\end{array}\right]$ considered in the example below. 


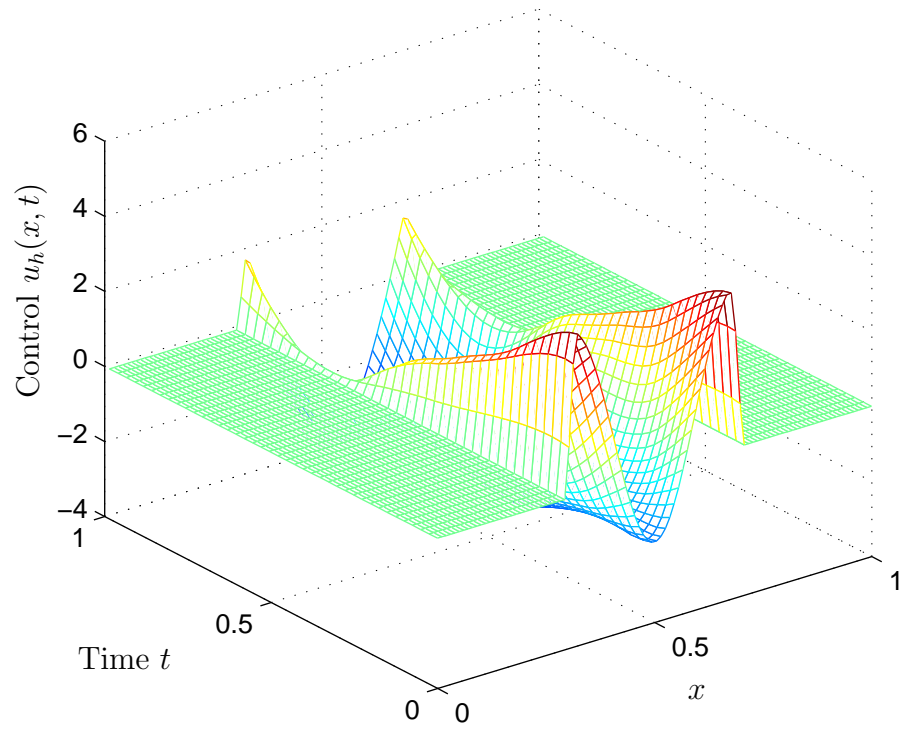

Figure 4: The approximation $u_{h}$ with the initial state $q_{0}(x)=x^{5}(1-x)^{5}, q_{1}(x)=-q_{0}(x)$ and the control time $\tau=1$.

\section{Acknowledgments}

The authors are partially supported by the bilateral project 19600SJ, Grant 206/2009 of ANCS (Romania). The second author is partially supported by Grant MTM2008-03541 funded by MICINN (Spain).

\section{References}

[1] G. BAKER, Error estimates for finite element methods for second order hyperbolic equations, SIAM J. Numer. Anal., 13 (1976), pp. 564-576.

[2] C. Bardos, G. Lebeau, And J. Rauch, Sharp sufficient conditions for the observation, control, and stabilization of waves from the boundary, SIAM J. Control Optim., 30 (1992), pp. 1024-1065.

[3] S. Brenner And L. Scott, The mathematical theory of finite element methods, vol. 15 of Texts in Applied Mathematics, Springer-Verlag, New York, 1994.

[4] G. Chen, Control and stabilization for the wave equation in a bounded domain, SIAM J. Control Optim., 17 (1979), pp. 66-81.

[5] S. Ervedoza And E. Zuazua, Hilbert uniqueness method and regularity: Applications to the order of convergence of discrete controls for the wave equation, (2009). Private communication.

[6] S. Ervedoza And E. ZuAzuA, Uniformly exponentially stable approximations for a class of damped systems, J. Math. Pures Appl. (9), 91 (2009), pp. 20-48.

[7] R. Glowinski, C. H. Li, And J.-L. Lions, A numerical approach to the exact boundary controllability of the wave equation (i). dirichlet controls: Description of the numerical methods, Japan J. Appl. Math., 7 (1990), pp. 1-76. 
[8] R. Glowinski And J.-L. Lions, Exact and approximate controllability for distributed parameter systems, Acta Numer., (1996), pp. 159-333.

[9] A. Haraux, Une remarque sur la stabilisation de certains systèmes du deuxième ordre en temps, Portugal. Math, 46 (1989), pp. 245-258.

[10] K. Ito, K. Ramdani, And M. Tucsnak, A time reversal based algorithm for solving initial data inverse problems, Discrete Contin. Dyn. Syst. Ser. S., (2010). to appear.

[11] J.-L. Lions, Contrôlabilité exacte, perturbations et stabilisation de systèmes distribués. Tome 1, vol. 8 of Recherches en Mathématiques Appliquées, Masson, Paris, 1988. With a chapter by E. Zuazua, and a chapter by C. Bardos, G. Lebeau and J. Rauch.

[12] K. LIU, Locally distributed control and damping for the conservative systems, SIAM J. Control Optim., 35 (1997), pp. 1574-1590.

[13] A. Quarteroni And A. Valli, Numerical approximation of partial differential equations, vol. 23 of Springer Series in Computational Mathematics, Springer-Verlag, Berlin, 1997.

[14] R. Rebarber And G. Weiss, An extension of Russell's principle on exact controllability, in Proc. of the Fourth ECC, 1997. CD-ROM.

[15] D. Russell, Exact boundary value controllability theorems for wave and heat processes in star-complemented regions, in Differential games and control theory (Proc. NSF-CBMS Regional Res. Conf., Univ. Rhode Island, Kingston, R.I., 1973), Dekker, New York, 1974, pp. 291-319. Lecture Notes in Pure Appl. Math., Vol. 10.

[16] — Controllability and stabilizability theory for linear partial differential equations: recent progress and open questions, SIAM Rev., 20 (1978), pp. 639-739.

[17] G. Strang And G. J. Fix, An analysis of the finite element method, Prentice-Hall Inc., Englewood Cliffs, N. J., 1973. Prentice-Hall Series in Automatic Computation.

[18] M. Tucsnak And G. Weiss, How to get a conservative well-posed linear system out of thin air. Part II: Controllability and stability, SIAM J. Control Optimization, 42 (2003), pp. 907-935.

[19] M. Tucsnak And G. WeIss, Observation and control for operator semigroups, Birkhäuser Advanced Texts: Basler Lehrbücher. [Birkhäuser Advanced Texts: Basel Textbooks], Birkhäuser Verlag, Basel, 2009.

[20] G. Weiss And M. TuCsnak, How to get a conservative well-posed linear system out of thin air. Part I: Well-posedness and energy balance, ESAIM Control Optim. Calc. Var., 9 (2003), pp. 247-274.

[21] E. Zuazua, Propagation, observation and control of waves approximated by finite difference methods, SIAM Rev., 47 (2005), pp. 197-243. 